\title{
Fibre Laser Using a Microchannel Based Loss Tuning Element for Refractive Index Sensing
}

\author{
Chengbo Mou, Kaiming Zhou, Edward Davies, Lin Zhang, and Ian Bennion \\ Photonics Research Group, Aston University, Birmingham, UK, B4 7ET
}

\begin{abstract}
We have proposed and demonstrated a fibre laser system using a microchannel as a cavity loss tuning element for surrounding medium refractive index (SRI) sensing. A $\sim 6 \mu \mathrm{m}$ width microchannel was created by femtosecond $(f s)$ laser inscription assisted chemical etching in the cavity fibre, which offers a direct access to the external liquids. When the SRI changes, the microchannel behaves as a loss tuning element, hence modulating the laser cavity loss and output power. The results indicate that the presented laser sensing system has a linear response to the SRI with a sensitivity in the order of $10^{-5}$. Using higher pump power and more sensitive photodetector, the SRI sensitivity could be further enhanced.
\end{abstract}

Key words: femtosecond laser, microchannel, fibre laser, refractive index sensor

\section{INTRODUCTION}

Optical fibre based sensors have been demonstrated as good candidates for various sensing applications due to their intrinsic merits compared to the electronic sensors. Recently, optical fibre sensors for SRI based bio/chemical sensing have received more attention. Conventional UV inscribed in-fibre Bragg grating (FBG) devices can not be applied as SRI sensors, because mode coupling only occurs between the core modes. Therefore, only modified FBG structures have been proposed for SRI sensing ${ }^{1-3}$. Majority of such structure modifications involve chemical etching to remove the fibre cladding completely, which seriously degrades the device strength. Alternatively, devices that can couple core mode to cladding mode, such as long period fibre gratings (LPFGs) ${ }^{4}$ and excessively tilted fibre gratings (ETFGs) ${ }^{5}$ provide high SRI sensitivity without the need for structure modification. Recently, femtosecond $(f s)$ laser processing technology has attracted more attention and been regarded as one of the most efficient way to inscribe microstructures onto silica materials and optical fibres. Very recently, $f_{s}$ laser inscription assisted chemical etching method has been developed to create microchannel in normal and chirped FBGs to introduce SRI sensitivity to the structures for SRI sensing ${ }^{6}$.

However, all these techniques are passive devices based using expensive apparatus, such as external broadband source and optical spectrum analyser for signal demodulation. We report here a novel and simple laser sensor system using intracavity microchannel for SRI sensing. Only low cost photodetector is required for signal demodulation. The proposed active laser sensor shows an SRI sensitivity of $\sim 10^{-5}$. In comparison with those cladding removal grating sensors, this microchannel based laser sensor system has maintained good mechanical strength. The low-cost demodulation, high SRI sensitivity and robustness could make this laser sensor system as an ideal candidate device for in-fibre probe chemical/biological sensing applications.

20th International Conference on Optical Fibre Sensors, edited by Julian Jones, Brian Culshaw, Wolfgang Ecke, José Miguel López-Higuera, Reinhardt Willsch, Proc. of SPIE Vol. 7503, 75035D (C) 2009 SPIE · CCC code: 0277-786X/09/\$18 - doi: 10.1117/12.834749

Proc. of SPIE Vol. 7503 75035D-1 


\section{SYSTEM CONFIGURATION AND OPERATION PRINCIPLE}

\subsection{Operation principle}

The basic principle of laser oscillation is that the gain can overcome any cavity induced loss, thus emits out coherence laser light. The output laser power can vary with the change of any loss mechanism in the laser resonator and also the pump power. An introduction of a small microchannel in the laser cavity offers a direct access to the liquid type surrounding medium, hence tunes the laser cavity loss. When the microchannel is filled with liquids of different SRI, the laser output changes accordingly. This correlation provides a simple method to measure the SRI and its related chemical and biological properties.

\subsection{Fabrication of in-fibre microchannel}

The fabrication procedure of the in-fibre microchannel involves two major steps: (1) $f s$-laser inscribing the microchannel pattern in a standard telecom fibre (Corning SMF-28); (2) etching the fibre in a 5\% hydrofluoric acid (HF) solution for removal of the $f s$-laser processed area. The $f s$ laser used for in-fibre microchannel inscription is a $800 \mathrm{~nm}$ mode locked Ti:Sapphire solid state laser. A 100× objective lens was used to focus the laser pulses onto the fibre. The fibre was mounted on a four-dimension (three translations plus rotation) air-bearing translation stage, and the microchannel was inscribed by moving the fibre in its cross section direction to expose to $f_{s}$ beam. After the $f s$ processing, the fibre with the patterned microchannel was immersed in the HF solution. An ultrasonic bath was also employed to improve the penetration of HF into the microchannel area. This selective etching procedure assists in creating the microchannel as well as maintaining the structural integrity near the machined region. The microchannel was inspected by a microscope after the etching process. Fig. 1 shows the microchannel images in the fibre under a $20 \times$ oil immersion objective lens. The channel width is measured around $\sim 6 \mu \mathrm{m}$.

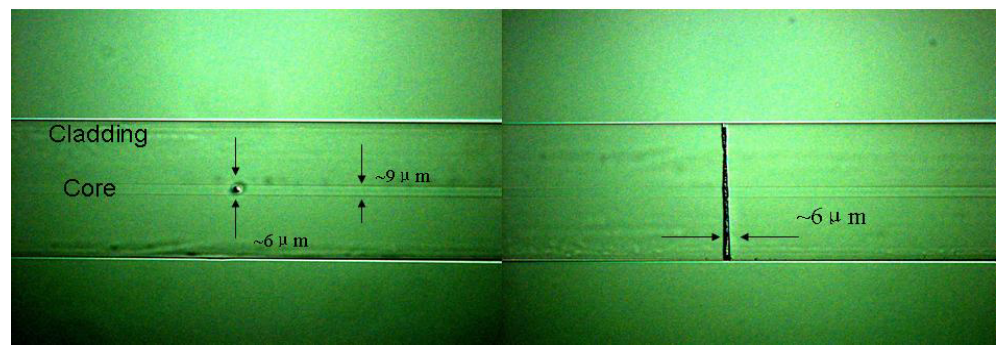

Figure.1 Microscope images of the in-fibre microchannel in SMF-28 fibre inspected by a $20 \times$ oil immersion objective lens

\subsection{Fibre laser setup}

The fibre laser structure used in this experiment is a standard linear cavity which is shown in Fig.2. A $1 \mathrm{~m}$ long Er/Yb co-doped fibre is used as the gain medium, a uniform FBG which has a reflectivity of $\sim 4 \mathrm{~dB}$ at $1551.4 \mathrm{~nm}$ is used as the output coupler of the laser, while a $3 \mathrm{~dB}$ coupler formed sagnac loop as the other high reflection cavity mirror. The laser is pump through a $980 / 1550$ WDM by a $975 \mathrm{~nm}$ laser diode. 


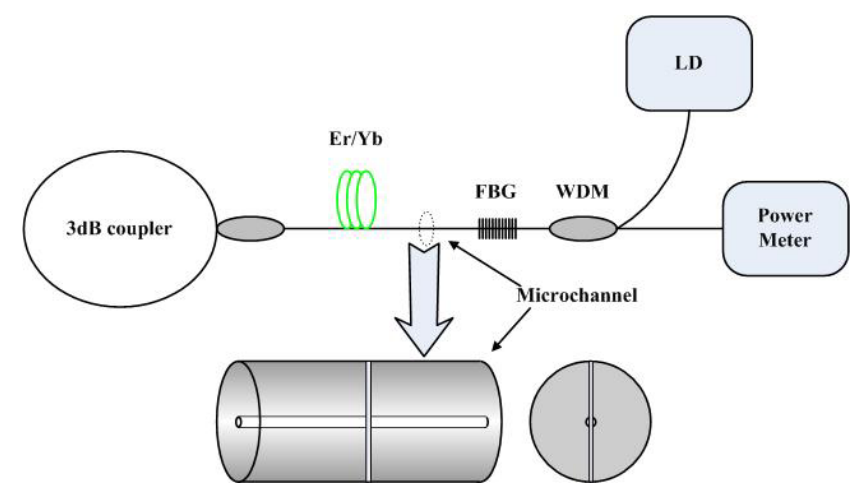

Figure.2 Schematic diagram of the proposed fibre laser with an in-cavity microchannel for SRI sensing.

\section{EXPERIMENTAL RESULTS}

To inspect the SRI response, we fixed the pump power to examine the output of the laser with SRI change in the microchannel. The laser output was monitored through a high speed optical power meter with a resolution of $0.2 \mu \mathrm{W}$. The part of fibre containing the microchannel was fixed from both sides on a V-grooved metal plate. We keep the microchannel part straight so that other effects, such as temperature, bending etc, could be eliminated. A series of certified refractive index gels were used as the surrounding RI media. After each measurement of the index gel, the microchannel part was cleaned carefully by acetone, methanol and deionised water until the laser output power recovered to its initial value in air. This is to make sure that there is no residual index gel left in the microchannel region.

The relative change in laser output power against SRI is shown in Fig.3(a) with a $73.14 \mathrm{~mW}$ pump power. As we can see, the laser output power has a linear response to the SRI from 1.37 to 1.452 . The equivalent sensitivity in this region is $\left(4.5 \times 10^{-5}\right) / 0.2 \mu \mathrm{W}$. We then changed the laser pump power and repeated the SRI measurement for each pump power and Fig.3(b) shows that the SRI sensitivity of the in-cavity microchannel laser sensor system increases with increasing pump power.
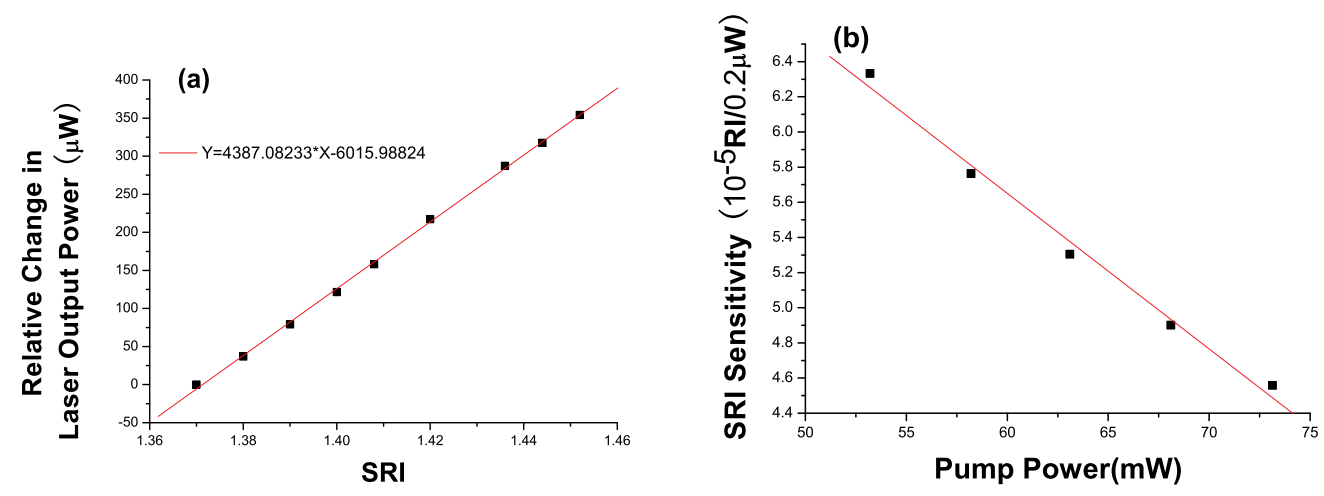

Figure.3 (a) Relative change in fibre laser output power against the SRI change when the pump power is fixed at $73.14 \mathrm{~mW}$; (b) SRI sensitivity against the pump power 


\section{DISCUSSION AND CONCLUSION}

Because the signal processing of this proposed laser sensor system mainly relies on the power detection, the stability of the laser cavity is very important. Any environmental fluctuation induced loss may degrade the sensor performance. The SRI sensitivity depends on the resolution of the detector, the pump power level and the size of the microchannel, therefore, higher SRI sensitivity can be achieved by using more sensitive detector, higher pump power and smaller size of the microchannel. However, the SRI may change if the environmental temperature changes, thus the system should be used for the applications involving insignificant thermal fluctuations, otherwise a compensation scheme should be employed to eliminate the temperature cross-sensitivity.

In summary, we have presented a low cost active SRI sensor system using a simple fibre laser configuration with an in-cavity microchannel. The microchannel was simply fabricated using the fs-laser inscription assisted HF etching and the small size of the microchannel will not affect the strength of the fibre, thus is more ideal in comparison with many reported passive FBG based SRI sensors whose cladding layers have been removed. Another advantage of this system is that the sensing signal can be demodulated using simple and low-cost photodiode.

\section{ACKNOWLEDGEMENT}

The authors would like to thank Dr. Xuewen Shu for providing the gain fibre.

\section{REFERENCE}

1. A. Iadicicco, A. Cusano, A. Cutolo,R. Bernini, and M. Giordano, "Thinned fiber Bragg gratings as high sensitivity refractive index sensors," IEEE Photon. Technol. Lett., 16,1149-1151, 2004.

2. Agostino Iadicicco, Stefania Campopiano, Antonello Cutolo, Michele Giordano, and Andrea Cusano, "Refractive index sensor based on microstructured fiber Bragg grating” IEEE Photon. Technol. Lett., 17,1250-1252, 2005.

3. A. Iadicicco, S. Campopiano, A. Cutolo, M. Giordano and A. Cusano, "Self temperature referenced refractive index sensor by non-uniform thinned fiber Bragg gratings", Sens. Actuators B, 120, 231-237, 2006.

4. X.Shu, L.Zhang, and I Bennion, "Sensitivity characteristics of Long-Period Fiber Gratings", J.Lightwave Technol., 20,255-266, 2002.

5. K.Zhou, L.Zhang, X.Chen, and I.Bennion, "Optic sensors of high refractive-index responsivity and low thermal cross sensitivity that use fiber Bragg gratings of $>80^{\circ}$ tilted structures”, Opt. Lett, 31,1193-1195,2006

6. Hongyan Fu, Kaiming Zhou, Pouneh Saffari, Chengbo Mou, Lin Zhang, Sailing He and Ian Bennion, ",Microchanneled Chirped Fiber Bragg Grating Fromed by Femtosecond Laser-Aided Chemical Etching for Refractive Index and Temperature Measurements” IEEE Photon. Technol. Lett, 20,1609-1611,2008 